\title{
Minitrack Introduction - Knowledge and Innovation Management: connecting theory to practice to business performance
}

\author{
Marianne Gloet \\ University of Melbourne \\ marianne.gloet@unimelb.edu.au
}

\author{
Danny Samson \\ University of Melbourne \\ d.samson@unimelb.edu.au
}

The fundamental role of knowledge in acquiring and maintaining competitive advantage emphasizes the need for effective and strategic knowledge and innovation management (KIM) in organizations. When effective and reliable methods drive approaches to KIM, this in turn supports the integration of valuecreating activities into organizational processes and increases an organization's potential to achieve innovation performance and business competitiveness.

The ultimate aim of knowledge management and innovation work is to have a positive impact on outcomes, namely business or organisational performance. Business performance can be broader than simply achieving profits and can indeed include environmental or social aspects of organisational impact and outcomes. We seek papers that either present conceptual frameworks or data/ evidence of the connections between knowledge and innovation management strategies and actions, and the broadlybased outcomes of these.

The first paper, by Good, Halinkski and Boekhorst, highlights some significant challenges surrounding positive knowledge management behaviors among employees in modern knowledge economies. Understanding how knowledge is managed between employees can bring significant value to organizations because positive knowledge management behaviors have a positive impact on an organization's competitive advantage. One way in which organizations have sought to facilitate more positive knowledge management behaviors is by creating 'fun' opportunities that enable employees to connect with others outside of their immediate group. Drawing from social exchange theory, this research develops a mediated-moderation model that examines the direct and indirect effects of participation in fun activities on three knowledge management behaviors (i.e., knowledge sharing, knowledge hiding, knowledge manipulating) and investigates the mediating role of positive affect and the moderating role of citizenship pressure on these relationships. Results highlight the importance of positive affect by showing the effects of participation in fun activities on knowledge management behaviors is dependent on whether or not participation in fun activities leads to positive affect. Data also shows citizenship pressure moderates the direct relationship between participation in fun activities and knowledge manipulating, as well as the indirect relationship between participation in fun activities and both knowledge sharing and hiding. These results highlight the theoretical and practical importance of both positive affect and citizenship pressure in understanding the dynamic relationship between workplace fun and knowledge management.

The second paper, by Zhao et. al. focuses on corporate strategy in accounting and examines the knowledge-transfer merger and acquisition (M\&A) data of listed companies from 2011 to 2016 in China. Regression analysis is used to construct a mediating effect model to determine whether business strategy is associated with knowledge-transfer M\&A by taking innovation level as a mediator variable. The study examined the impact of business strategy on innovation level, and the extent to whether prospector or defender strategies are more likely to have a higher innovation level. The research indicates that companies adopting prospector business strategies are more inclined to utilize knowledge-transfer M\&A than those that adopt defender business strategies, which is consistent with prior research suggesting that prospectors are usually innovative enterprises which are more radical while defenders are more cautious about innovative behaviors. The research also found that business strategies are associated with innovation level and prospectors are more likely to have a higher innovation level. Finally, the study indicates that the business strategy influences knowledge-transfer M\&A through the mediator variable of innovation level. The paper provides evidence that differences in the choice of business strategy is an underlying determinant of the likelihood of knowledge-transfer M\&As and innovation level. Secondly, we construct a comprehensive, theory-based mediating effect model 
of business strategy, innovation level and knowledgetransfer M\&As which reveals the internal relationship among them. It broadens the application of corporate strategy in accounting research.

Finally, the paper by Gloet and Samson extends exploratory research on the contribution of knowledge and innovation management (KIM) to innovation and sustainability activities across a number of small to medium size Australian food and beverage exporters in Australia as part of a longitudinal research project. Recent trends in sustainable supply chain management (SSCM) in global supply chains sees a greater focus on achieving more social and transformational forms of sustainability, rather than traditional economic or environmental approaches. Applying a framework of sustainability-oriented innovation, analysis of eight case study organizations revealed that innovation practices across these firms largely reflected an economic focus on sustainability, followed by some activities in the environmental domain. However, more transformative forms of innovation, such as those addressing social/community concerns, were lagging. Although further research is recommended, we offer some propositional speculation on why successful SMEs with a strong reputation for innovation are still driven predominantly by financial considerations. 